\title{
Two siblings with Bardet-Biedl syndrome caused by mutations in BBS10: the first case identified in Korea
}

\author{
Sung Chul Yoon, Hye Jin Lee, Jung Min Ko ${ }^{1,2, *}$, Hee Gyung Kang ${ }^{1,2}$, Hae Il Cheong ${ }^{1,2}$, Hyeong Gon Yu ${ }^{3}$, and Jae Hyung Kim ${ }^{4}$ \\ ${ }^{1}$ Department of Pediatrics, Seoul National University College of Medicine, Seoul, Korea \\ ${ }^{2}$ Research Coordination Center for Rare Diseases, Seoul National University Hospital, Seoul, Korea \\ ${ }^{3}$ Department of Opthalmology, Seoul National University College of Medicine, Seoul, Korea \\ ${ }^{4}$ Department of Opthalmology, Chungbuk National University Hospital, Cheongju, Korea
}

Bardet-Biedl syndrome (BBS) is a rare ciliopathy generally inherited with an autosomal recessive pattern. BBS is characterized by 6 primary features namely retinal dystrophy, obesity, postaxial polydactyly, renal dysfunction, learning difficulties, and hypogonadism and a wide range of secondary features. To date, mutations in 16 genes have been identified as causative factors for BBS. Among them, the BBS1 and BBS10 genes are major disease-causing genes, and each of these gene mutations presents in more than $20 \%$ of all BBS patients. Genotype-phenotype correlations have not been observed in BBS, and there can be phenotypic overlap between BBS and other ciliopathies. In Korea, no molecular, genetically confirmed case of BBS has been reported to date. Herein, we describe the case of the first Korean siblings with BBS resulting from 2 BBS10 gene mutations who showed typical clinical phenotypes, including retinal dystrophy, obesity, intellectual disability, cystic tubular disease, and postaxial polydactyly.

Key words: Bardet-Biedl syndrome, Retinitis pigmentosa, Polycystic kidney diseases, Polydactyly, BBS10.

\section{Introduction}

Bardet-Biedl syndrome (BBS, OMIM\#209900) is a rare genetic ciliopathy with a prevalence of 1 in 160,000 in Europeans and 1 in 13,500 in some Arab populations [1]. The primary clinical features of BBS include retinal dystrophy, obesity, postaxial polydactyly, renal dysfunction, learning difficulties, and hypogonadism $[1,2]$. In addition, a wide range of secondary features namely cataracts/strabismus, ataxia, hypertonia, diabetes mellitus, dental crowding/hypodontia, anosmia, auditory deficiencies, and Hirschsprung's disease are also observed [3]. Traditionally, BBS is clinically diagnosed when a patient shows either 4 primary features or 3 primary and 2 secondary features [1,4]. In addition to the pleiotropic symptoms and signs observed clinically in multiple organ systems, the genetic background of BBS is quite heterogeneous with significant inter-and intrafamilial variations. The inheritance pattern of BBS is traditionally considered to be autosomal recessive [1]. To date, mutations in 16 genes have been identified as causative of BBS. All of these genes account for approximately $80 \%$ of clinically diagnosed cases of BBS. Among them, BBS1 and BBS10 are the 2 main genes involved in $B B S$, and each of these gene mutations presents in more than $20 \%$ of the cases [1].

To date, 7 clinically diagnosed cases of BBS have been reported

Received: 23 April 2014, Revised: 20 May 2014, Accepted: 5 June 2014, Published: 30 June 2014

${ }^{*}$ Corresponding author: Jung Min Ko, M.D., Ph.D.

Department of Pediatrics, Seoul National University Children's Hospital, Seoul National University College of Medicine, 101 Daehak-ro, Jongno-gu, Seoul 110-769, Korea.

Tel: +82-2-2072-3570, Fax: +82-2-743-3455, E-mail: jmko@snu.ac.kr

Conflict of interest: We declare that we do not have any conflicts of interests.

(c) This is an open-access article distributed under the terms of the Creative Commons Attribution Non-Commercial License (http://creativecommons.org/licenses/by-nc/3.0/) which permits unrestricted non-commercial use, distribution, and reproduction in any medium, provided the original work is properly cited.

(c) Copyright 2014 by the Korean Society of Medical Genetics

www.e-kjgm.org 
in Korea [5,6], but there is no molecularly confirmed BBS case with mutations identified in the causative genes. Herein, we describe the case of the first Korean siblings with BBS resulting from 2 BBS 10 mutations who manifested typical clinical phenotypes, including retinal dystrophy, obesity, intellectual disability, cystic tubular disease, and postaxial polydactyly.

\section{Case}

A 17.5-year-old Korean boy visited the Seoul National University Children's Hospital (Seoul, Korea) because of progressive worsening of visual acuity. He was born with a weight of 3.25 $\mathrm{kg}$ at $41+5$ weeks of gestation by cesarean section and was the first child born to healthy and nonconsanguineous parents. At the age of 4 days, he showed abdominal distension and was diagnosed with Hirschsprung's disease at the regional hospital. Correction surgery for Hirschsprung's disease was performed. At the age of 18 months, he also underwent surgery for correcting the postaxial polydactyly of the right foot. His developmental history showed that he was able to walk alone at the age of 18 months, and borderline mental retardation was diagnosed after a neuropsychological evaluation. At the age of 6 years, nystagmus was observed, and pigmentary retinopathy was firstly detected by fundoscopic examination. During the annual follow-up, his visual acuity had worsened and he was referred to our hospital. His best-corrected visual acuity was $20 / 320$ (plano $-1.0 \times 180^{\circ}$ ) in his right eye and $20 / 400\left(+1.0-5.0 \times 180^{\circ}\right)$ in his left eye. Fundus examination showed the classic clinical triad of retinitis pigmentosa (i.e., arteriolar attenuation, bony spicules, and a waxy, pale disc) in both eyes (Fig. 1). Full-field electroretinography (ERG) and Goldmann visual field test demonstrated nondetectable ERG responses and concentric loss.
At the initial examination at our hospital, the patient's height and weight were $166 \mathrm{~cm}$ (10-25th percentile) and $87.25 \mathrm{~kg}$ (9597th percentile), respectively. He was obese, with a body mass index of 31.66 ( $>97$ th percentile). The scars from the previous surgery were visible on the abdomen and lateral side of the right fifth toe. He also showed secondary sexual characteristics, including axillary and pubic hair, pubertal-sized testicles, and a penile length of $5 \mathrm{~cm}$.

Laboratory examinations yielded normal results for blood cell counts and aminotransferase levels. His blood urea nitrogen level was $15 \mathrm{mg} / \mathrm{dL}$ and serum creatinine level was $1.25 \mathrm{mg} /$ $\mathrm{dL}$. Proteinuria or glycosuria was not observed. Fasting plasma lipid profiles were normal. The results of a 75-g oral glucose tolerance test showed fasting plasma glucose and insulin levels of $84 \mathrm{mg} / \mathrm{dL}$ and $10.9 \mu \mathrm{lU} / \mathrm{mL}$, respectively, and 2-h glucose and insulin levels of $96 \mathrm{mg} / \mathrm{dL}$ and $43.8 \mu \mathrm{lU} / \mathrm{mL}$, respectively. The levels of follicle-stimulating hormone, luteinizing hormone, and testosterone were normal for his age.

The younger brother of the patient visited the clinical genetics clinic of our hospital at the age of 6.5 years. Regarding his past history, pelviectasia of both kidneys was detected by prenatal ultrasonography at the 25th week of gestation. He was born at $38+2$ weeks of gestation with a birth weight of $3.18 \mathrm{~kg}$ (25th50th percentile). The findings of the initial examination included no gross anomaly. Kidney ultrasonography revealed mild enlargement with several small cortical cysts in the right kidney and diffuse increased echogenicity and poor corticomedullary differentiation in both kidneys, suggesting infantile polycystic kidney disease (Fig. 2). The follow-up ultrasonographic findings revealed no visible changes in the mild enlargement, several small cortical cysts, and diffuse increased echogenicity in both kidneys.

At the age of 6.5 years, his height and weight were $116.6 \mathrm{~cm}$

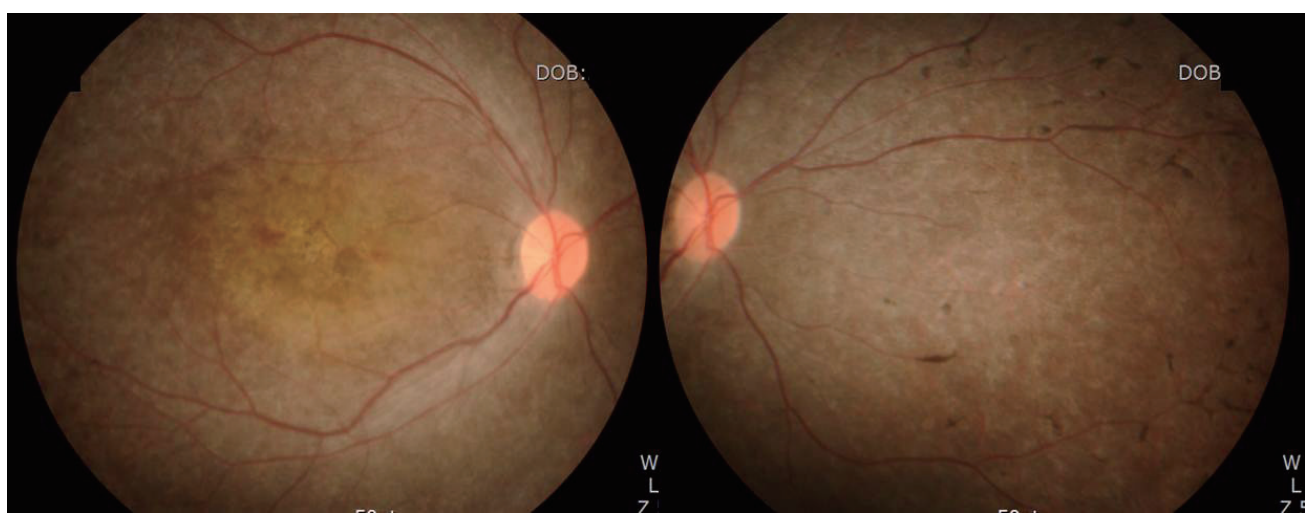

Fig. 1. Fundoscopic examination demonstrates the triad of retinitis pigmentosa, i.e., arteriolar attenuation, bony spicules, and a waxy, pale disc in both eyes. 


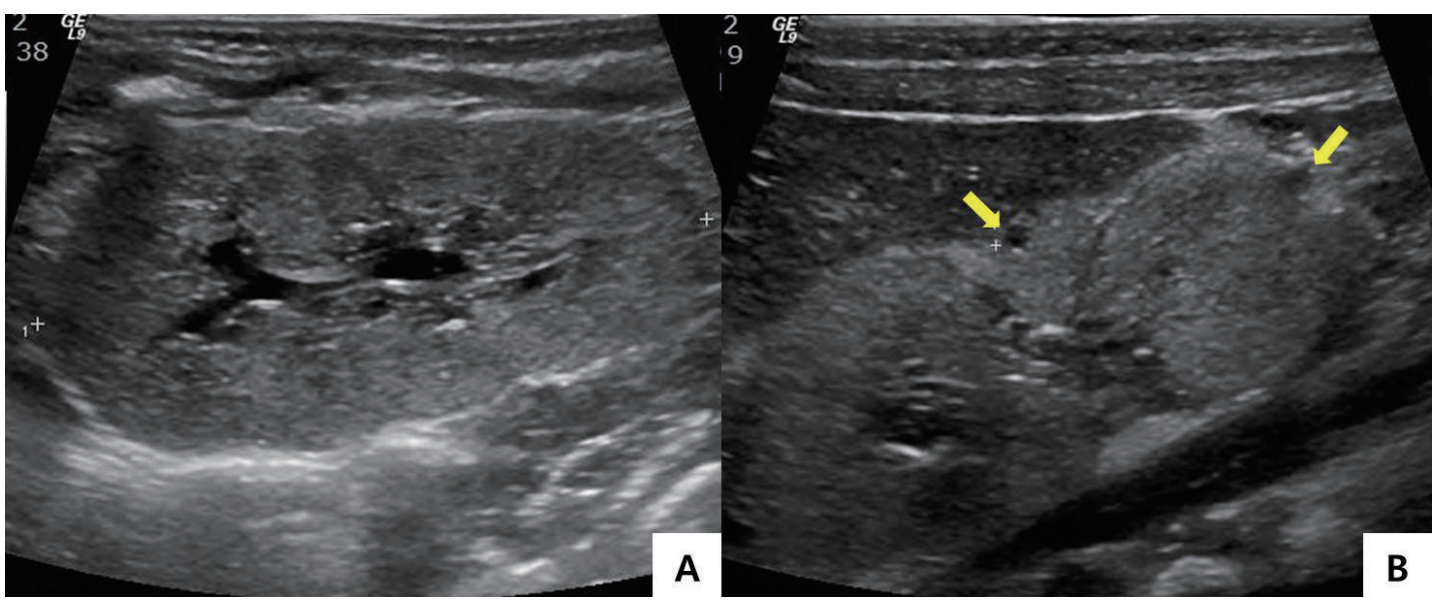

Fig. 2. Ultrasonography images. (A) Mild enlargement, diffuse increased echogenicity, and poor corticomedullary differentiation are observed in both kidneys. (B) Several small cortical cysts are present in the right kidney (arrows).

(25th-50th percentile) and $30.0 \mathrm{~kg}$ (90th-95th percentile), respectively. He was obese, with a body mass index of 22.07 (>97th percentile). He also exhibited mild developmental delay, strabismus, and decreased visual acuity at night. Pigmented retinopathy was suspected by fundoscopic examination. The findings of routine laboratory analyses of blood and urine were normal.

Familial BBS was suspected based on the family's medical history and the findings of physical examinations. The parents of these patients provided formal informed consent for peripheral blood sampling for genetic analyses. Considering that mutations were identified in the BBS1 and BBS10 genes in more than 40\% of all BBS cases, we first performed direct sequencing analysis of these 2 genes in the siblings. Two heterozygous mutations were identified in BBS10: a novel insertion, c.235_236insA (p.T78Nfs*17), in exon 2 and a previously reported missense mutation, c.530A>G (p.Y177C), in exon 2 [7]. No mutation was identified in BBS1. The mother and the father harbored the c.235_236insA and c.530A>G mutations, respectively (Fig. 3).

\section{Discussion}

BBS is a rare genetic malformation syndrome that belongs to the group of ciliopathies. Because of the extensive roles of primary cilia in the development of many organs, including the kidneys, brain, liver, eyes, and bones, a broad spectrum of phenotypes is observed in ciliopathies. In addition to BBS, the Alstrom, Joubert, McKusick-Kaufman, Jeune, and MeckelGruber syndromes also belong to the group of ciliopathies. Phenotypes, causative genes, and mutations overlap between ciliopathies to a certain extent [2].

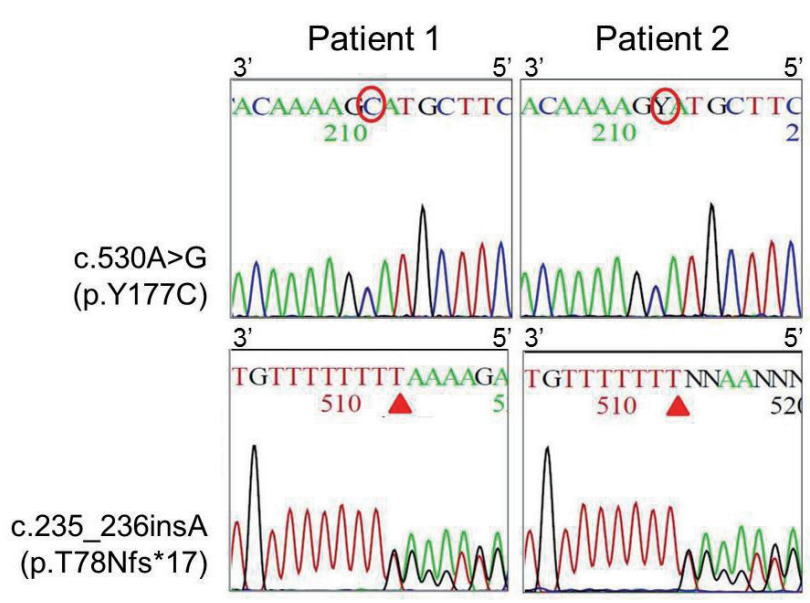

Fig. 3. Partial sequence of the $B B S 10$ gene showing the mutations detected in the siblings. A heterozygous $A>G$ mutation at nucleotide 530 causes an amino acid change from tyrosine to cysteine (c.530A $>\mathrm{G}$, p.Y177C). A novel heterozygous insertion of $A$ between nucleotides 235 and 236 leads to premature termination of the synthesis of the BBS10 protein via the creation of a frameshift (c.235_236insA, p.T78Nfs*17).

The primary cilium comprises the axoneme, basal body, and transition zone. The BBSome is a component of the basal body and is a complex consisting of 7 BBS-associated proteins, BBS1, BBS2, BBS4, BBS5, BBS7, BBS8, and BBS9. The chaperonin complex is the complex consisting of 3 BBS proteins, BBS6, BBS10, and BBS12 $[1,8,9]$, and coordinates the assembly of the BBSome, which interacts with BBS3 to organize and maintain the cilium. The first causative gene for BBS (BBS1) was identified in 2002 [10], and a total of 16 disease-causing BBS genes have been discovered to date [1]. Mutations of each BBS gene lead to problems with the structure and function of the cilia [1]. Among them, $B B S 1$ and $B B S 10$ are major causative genes of this disease. Our patients had mutations in BBS10. The previously reported 
mutation, p.Y177C, is located in the proximal intermediate domain, which is a critical binding site for BBS6 and BBS12 during the formation of the chaperonin complex $[10,11]$. The novel insertion mutation identified in this study, c.235_236insA, causes premature termination of BBS10 synthesis by a frameshift mutation, p.T78Nfs*17. Although no definite genotypephenotype correlations have been reported for BBS [1], several previous studies have suggested that specific ocular problems and severe digital problems may be linked to mutations in a certain gene $[1,12,13]$. Previous studies showed that BBS1O mutations were identified frequently in patients with Meckeltype cystic kidneys, including severely lethal cases [14], and tended to be associated with more severe visceral adiposity and higher insulin resistance than did mutations in the other genes [15]. In this study, the younger brother showed cystic kidneys from the fetal period. However, the elder brother did not have renal dysfunction until the age of 17.5 years, and polydactyly and Hirschsprung's disease were only present in the elder brother. These discrepancies in clinical phenotypes between the siblings suggest the variable expression of BBS10.

BBS shows pleiotropic phenotypes involving multiple organ systems. BBS is usually diagnosed during childhood, when the patient starts to develop visual problems due to retinal dystrophy [1]. Night blindness is generally obvious by the age of 7 to 8 years, and legal blindness manifests itself at the mean age of 15.5 years in children with BBS [3]. Significant weight gain begins in the neonatal period and becomes a chronic problem. The adipose tissue is widespread in children and adolescents but becomes most prominent in the trunk and proximal limbs in adults [3]. Both structural and functional renal diseases have been associated with BBS. Progressive renal dysfunction often occurs in BBS and can lead to end-stage renal disease, requiring kidney transplantation in up to 10\% of affected patients [3]. In particular, renal involvement in BBS is thought to be an important factor for the prognosis of this condition. Therefore, routine checking of blood pressure and annual renal examination involving laboratory and imaging studies need to continue in these 2 patients.

To the best of our knowledge, this is the first study reporting the case of 2 siblings with BBS with compound heterozygous mutations in BBS10. The siblings had retinal dystrophy, obesity, intellectual disability, cystic tubular disease, and postaxial polydactyly, which are typical clinical phenotypes of BBS. Although BBS is a rare genetic disease, it is inherited in an autosomal recessive manner and can recur in patients' siblings. Regarding the concerns about multiple-organ complications, a multidirectional approach for accompanying medical problems and life-long surveillance of chronic complications are required for managing this pleiotropic condition effectively.

\section{Acknowledgements}

We express our gratitude to the patient and his family for their participation in this study. This study was supported by a grant of NRF-2012R1A1A3001588 from the Korean Ministry of Science, ICT and Future Planning.

\section{References}

1. Forsythe E, Beales PL. Bardet-Biedl syndrome. Eur J Hum Genet 2013;21:8-13.

2. Badano JL, Mitsuma N, Beales PL, Katsanis N. The ciliopathies: an emerging class of human genetic disorders. Annu Rev Genomics Hum Genet 2006;7:125-48.

3. Forsythe E, Beales PL. Bardet-Biedl syndrome 2003 [Updated 2014]. [http://www.ncbi.nlm.nih.gov/books/NBK1363/]

4. Beales PL, Katsanis N, Lewis RA, Ansley SJ, Elcioglu N, Raza J, et al. Genetic and mutational analyses of a large multiethnic Bardet-Biedl cohort reveal a minor involvement of BBS6 and delineate the critical intervals of other loci. Am J Hum Genet 2001;68:606-16.

5. Jeon PK, Ohn YH, Park JO, Kim CH. A case of laurence-moon-biedl syndrome. Korean J Pediatr Gastroenterol Nutr 2003;6:78-83.

6. Park LK, Lee DH, Moon C, Kim EM. A case of laurence moon-bardet biedl syndrome with chronic renal failure. J Korean Soc Pediatr Nephrol 1998:2:200-3.

7. Harville HM, Held S, Diaz-Font A, Davis EE, Diplas BH, Lewis RA, et al. Identification of 11 novel mutations in eight BBS genes by highresolution homozygosity mapping. J Med Genet 2010;47:262-7.

8. Jin H, Nachury MV. The BBSome. Curr Biol 2009;19:R472-3.

9. Jin H, White SR, Shida T, Schulz S, Aguiar M, Gygi SP, et al. The conserved Bardet-Biedl syndrome proteins assemble a coat that traffics membrane proteins to cilia. Cell 2010;141:1208-19.

10. Mykytyn K, Nishimura DY, Searby CC, Shastri M, Yen HJ, Beck JS, et al. Identification of the gene (BBS1) most commonly involved in BardetBiedl syndrome, a complex human obesity syndrome. Nat Genet 2002;31:435-8.

11. Stoetzel $C$, Laurier V, Davis EE, Muller J, Rix S, Badano JL, et al. BBS10 encodes a vertebrate-specific chaperonin-like protein and is a major BBS locus. Nat Genet 2006;38:521-4.

12. Riise $R$, Tornqvist $K$, Wright AF, Mykytyn $K$, Sheffield VC. The phenotype in Norwegian patients with Bardet-Biedl syndrome with mutations in the 
BBS4 gene. Arch Ophthalmol 2002;120:1364-7.

13. Héon E, Westall C, Carmi R, Elbedour K, Panton C, Mackeen L, et al. Ocular phenotypes of three genetic variants of Bardet-Biedl syndrome. Am J Med Genet A 2005;132A:283-7.

14. Putoux A, Mougou-Zerelli S, Thomas S, Elkhartoufi N, Audollent S, Le
Merrer M, et al. BBS10 mutations are common in 'Meckel'-type cystic kidneys. J Med Genet 2010;47:848-52.

15. Feuillan PP, Ng D, Han JC, Sapp JC, Wetsch K, Spaulding E, et al. Patients with Bardet-Biedl syndrome have hyperleptinemia suggestive of leptin resistance. J Clin Endocrinol Metab 2011;96:E528-35. 